\title{
Percepções dos educadores sobre a inclusão na educação pré-escolar: o papel da experiência e das habilitações
}

\author{
Paulo César Azevedo Dias ${ }^{a}$ \\ Irene Maria Dias Cadime ${ }^{b}$
}

\section{Resumo}

Em Portugal, a extensão dos serviços de educação especial à educação pré-escolar é relativamente recente. São também escassos os estudos que avaliam as percepções dos educadores de infância sobre as práticas inclusivas. Neste trabalho, pretendemos avaliar as percepções dos educadores de infância sobre a inclusão. Recorreu-se a uma amostra de 118 educadores de infância portugueses, a quem foi administrado o Inventário de Inclusão. Os resultados indicam que a implementação de práticas inclusivas está relacionada com a percepção de apoio e utilização anterior de práticas inclusivas. Educadores mais novos, com menos experiência letiva e com menos experiência na aplicação de práticas inclusivas possuem mais crenças sobre a inclusão. Educadores mais velhos e que trabalham mais tempo com crianças com necessidades educativas especiais percebem como melhores os efeitos da inclusão. Esses resultados são discutidos à luz da literatura existente, apresentando-se também implicações para a prática educativa e para a investigação.

Palavras-chave: Inclusão. Pré-escolar. Percepções. Educadores de infância.

\section{Introdução}

A idade em que as crianças frequentam a educação pré-escolar ${ }^{1}$ corresponde a um período crítico para o desenvolvimento das suas competências, nos domínios cognitivo, social e emocional, sendo, por isso, de máxima importância que as práticas implementadas neste ciclo de ensino maximizem as oportunidades de desenvolvimento das mesmas, independentemente do nível socioeconómico ou

\footnotetext{
a Universidade Católica Portuguesa - UCP Braga, Portugal.

b Universidade do Minho - UMinho. Braga, Portugal.

1 Neste artigo, referimo-nos à educação pré-escolar enquadrada no sistema educativo português, sendo legalmente estipulado que esta se destina a crianças com idades compreendidas entre os três anos e a entrada na escolaridade obrigatória que, em Portugal, ocorre por volta dos seis anos de idade (PORTUGAL, 1997a).
} 
classe social de origem de cada criança. Esta maximização de oportunidades assume especial relevância no caso de crianças com necessidades educativas especiais (NEE). Para além de se constituir como um contexto preferencial para o fomento da natural interação com as outras crianças da mesma faixa etária, a educação pré-escolar permite às crianças e famílias aceder a serviços e recursos essenciais para potenciar o seu desenvolvimento.

Sendo relativamente recente a extensão, em termos formais, dos serviços de educação especial ${ }^{2}$ à educação pré-escolar, legalmente prevista apenas em 2008, tem-se assistido a um progressivo investimento na formação de profissionais e dotação de recursos nas escolas deste nível de ensino, numa tentativa de as preparar para enfrentar os novos desafios relacionados com a inclusão de crianças com necessidades educativas especiais. Contudo, são escassos os estudos que avaliam as percepções dos educadores do pré-escolar sobre as práticas inclusivas no contexto português. Com base numa revisão apurada da literatura e com a recolha de informação a ser efetuada com um instrumento abrangente e sensível a este novo paradigma de Escola, pretendemos com este trabalho explorar o que os educadores pensam sobre a implementação da inclusão nas suas escolas e investigar a existência de variáveis que expliquem as diferenças de percepção. Espera-se que os resultados obtidos contribuam para uma reflexão mais informada sobre o estado da arte e para a melhoria das respostas das escolas e da formação inicial e contínua dos educadores.

\section{Desafios da inclusão em contexto pré-escolar}

Como referem Bairrão e Vasconcelos (1997), a implementação da educação pré-escolar em Portugal reflete em grande medida, o percurso percorrido noutros países europeus, apesar de se verificar um significativo atraso na implantação das respostas públicas, comparativamente aos restantes países. Um salto qualitativo importante ocorre em 1997, com a criação de uma rede nacional de estabelecimentos de ensino pré-escolar, a definição de orientações para as características físicas dos estabelecimentos (DL n ${ }^{\circ}$ 147/1997) e a criação das orientações curriculares para a educação pré-escolar (PORTUGAL, 1997b). É também a partir desta altura que se começa a assistir à implementação progressiva de sistemas de avaliação e promoção da qualidade das instituições de educação pré-escolar e que surge um investimento gradual na formação e na melhoria das práticas dos educadores.

\footnotetext{
Devem entender-se por serviços de educação especial, os serviços que prestam apoios especializados "visando a criação de condições para a adequação do processo educativo às necessidades educativas especiais dos alunos com limitações significativas ao nível da atividade e da participação num ou vários domínios de vida, decorrentes de alterações funcionais e estruturais, de carácter permanente, resultando em dificuldades continuadas ao nível da comunicação, da aprendizagem, da mobilidade, da autonomia, do relacionamento interpessoal e da participação social" (PORTUGAL, 2008, p. 155).
} 
Embora não seja um nível de ensino obrigatório, o número de alunos que frequentam o ensino pré-escolar tem vindo a aumentar, sendo atualmente garantida a sua universalidade. Estatísticas oficiais indicavam que, em Portugal, no ano letivo 2013/2014, frequentavam a educação pré-escolar 265.414 crianças, das quais $2.450 \mathrm{com}$ necessidades educativas especiais (DGEEC, 2015). A entrada desta população nas instituições de educação pré-escolar é, contudo, um movimento recente. Apesar de Portugal ser um dos países signatários da Declaração de Salamanca (UNESCO, 1994), e de ter legislação para a inclusão de crianças na educação obrigatória desde o início dos anos 1990 (PORTUGAL, 1991), só em 2008 é que a legislação estende os serviços de educação especial ao ensino pré-escolar, definindo as medidas e os recursos aplicáveis a todas as escolas.

A investigação que explora os benefícios sociais da inclusão tem observado os seus efeitos em variáveis como a aceitação social, a rejeição social, as amizades, as competências sociais ou a vitimização dos alunos com necessidades educativas especiais (PRINCE; HADWIN, 2013). Por exemplo, Wiener e Tardif (2004) estudaram o efeito da inclusão de alunos com necessidades educativas especiais nas turmas regulares, nos seus sentimentos de amizade, solidão, autopercepção e competências sociais, por comparação com alunos que não eram incluídos. Os resultados mostraram que os alunos alvo de práticas de inclusão eram mais aceites pelos pares, tinham autopercepções académicas mais positivas, relações mais satisfatórias com os colegas e sentiam-se menos solitários (WIENER; TARDIF, 2004). Embora estes resultados tenham sido observados para níveis de escolaridade mais avançados, é possível que os benefícios da implementação de práticas inclusivas se possam estender ao pré-escolar. Estudos recentes mostram mesmo que intervenções focadas na promoção da inclusão junto dos pares parecem ter maior efeito em contexto pré-escolar do que em ciclos de escolaridade posteriores (DE BOER et al., 2014). Salvaguardando questões culturais, outros estudos apontam ainda os efeitos benéficos da experiência inclusiva em contexto pré-escolar nas competências sociais dos pares sem necessidades especiais, seja ao nível da agressão, comportamento antissocial, vitimação, competências sociais ou autocontrolo (OGELMAN; SECER, 2012). Percebe-se, portanto, que o contexto do pré-escolar é essencial para a intervenção precoce, mas também para uma sensibilização geral das crianças para as diferenças individuais e promoção da equidade (VASCONCELOS, 2005; DIAMOND et al., 2013). Para as crianças com necessidades especiais, a frequência da educação préescolar pode ser um recurso essencial para a estimulação das suas áreas fortes (que podem variar de criança para criança) e desenvolvimento de competências num contexto normativo; para as crianças sem necessidades especiais, pode ser um contexto rico para o desenvolvimento da compreensão e respeito pelas diferenças e de promoção de competências sociais (por exemplo, RUIJS; PEETSMA, 2009; HENNINGER IV; GUPTA, 2014). 
Além disso, a educação pré-escolar tem um papel muito importante no estabelecimento de uma relação positiva que envolva os pais na educação dos seus filhos. Se isso é importante em qualquer idade, é particularmente relevante nas idades mais precoces, apesar de alguns estudos apontarem a existência de um escasso envolvimento parental e pouca colaboração entre a escola e a família em contextos inclusivos (RODRIGUES et al., 2015).

Apesar do papel significativo que o ensino pré-escolar e que os educadores de infância podem ter no desenvolvimento integral das crianças que atendem, a investigação dirigida a este público, no que toca às suas percepções e práticas de inclusão, tem sido relativamente reduzida. Na parca investigação realizada a este nível no contexto português, o foco parece recair sobretudo na avaliação de necessidades e não na avaliação de programas ou intervenções para promover a inclusão educativa (CALHEIROS; GRAÇA; PATRÍCIO, 2014). Na literatura internacional, vão sendo já publicados diversos trabalhos que apresentam resultados de intervenções junto de crianças com Perturbações do Espectro do Autismo (por exemplo, HUNDERT; ROWE; HARRISON, 2014; SAINATO et al., 2015), perturbações desenvolvimentais ou do comportamento (por exemplo, PEARS et al., 2014), assim como programas dirigidos aos pares de crianças com necessidades especiais (por exemplo, DE BOER et al., 2014) que poderão ser adaptados para o nosso contexto. Numa altura em que os órgãos de aconselhamento governamental, tal como o Conselho Nacional de Educação, começam a chamar a atenção para a importância da educação na faixa etária que compreende os 0-3 anos (VASCONCELOS, 2013), importará discutir o estado da arte, as percepções e sentimento de competência dos educadores para promover a inclusão em idades precoces. Com base em evidências sólidas, acreditamos que será possível desenvolver respostas que permitam educação de qualidade para todos.

Os resultados da investigação com professores que lecionam em anos de escolaridade mais avançados parecem convergir em algumas linhas gerais: os professores tendem a reportar uma visão positiva da inclusão como modelo de inclusão social benéfica, tanto para alunos com, como sem necessidades educativas especiais (AVRAMIDIS; BAYLISS; BURDEN, 2000; HEIMAN, 2004; AVRAMIDIS; KALYVA, 2007; DIAS, 2015), mas manifestam preocupações sobre as condições necessárias para a sua implementação. Estas preocupações centram-se essencialmente sobre a preparação dos professores do ensino regular para responderem adequadamente aos desafios que os alunos com necessidades educativas especiais colocam, sobre o apoio necessário de outros professores especializados e dos diversos departamentos educativos, sejam eles a nível de escola ou da comunidade mais abrangente, e sobre a disponibilidade dos materiais e recursos de ensino necessários para atender às especificidades de cada aluno (por exemplo, HORNE; TIMMONS, 2009; 
BALLHYSA; FLAGLER, 2011; SÁNCHEZ; ABELLÁN, 2014; DIAS, 2015). Ballhysa e Flagler (2011) sumariam em quatro grandes grupos estas preocupações, observadas em professores a lecionar entre o $1^{\circ}$ e o $9^{\circ}$ ano de escolaridade: (a) falta de preparação adequada dos profissionais para trabalhar com alunos com necessidades educativas especiais em ambientes inclusivos; (b) falta de suporte administrativo, tal como turmas com tamanho reduzido ou carga horária ajustada, de forma a possibilitar mais tempo para o apoio às necessidades individualizadas dos alunos com necessidades educativas especiais; (c) falta de apoio de outros profissionais especializados, entre os quais, professores de educação especial; (d) falta de recursos materiais de apoio, tais como equipamentos ou materiais didáticos adaptados. Estas percepções sobre a falta/adequação de recursos podem exercer efeitos sobre as suas atitudes perante a inclusão que influenciam, em larga medida, a sua atuação posterior. As atitudes face à inclusão parecem ser influenciadas não só por variáveis sociodemográficas e variáveis relacionadas com a formação e experiência docente, tais como o sexo, a idade, o tempo de serviço ou a frequência de formação inicial ou avançada relacionada com a inclusão (AVRAMIDIS; NORWICH, 2002; LOREMAN; FORLIN; SHARMA, 2007; DE BOER; PIJL; MINNAERT, 2011; DIAS, 2015), mas também por variáveis de tipo psicológico. Por exemplo, o grau de confiança em ensinar alunos com necessidades educativas especiais parece encontrar-se estreitamente relacionado com atitudes mais positivas perante a inclusão (LOREMAN; FORLIN; SHARMA, 2007).

Os resultados de um estudo recente no contexto português, realizado com educadores do pré-escolar, mostraram que tais educadores possuem, na generalidade, atitudes positivas perante a inclusão de crianças com necessidades educativas especiais em escolas regulares, mas também que ter tido experiência prévia no ensino de turmas, as quais incluíam crianças com necessidades educativas especiais, estava associado a um nível inferior de intenções comportamentais, o que poderá diminuir a probabilidade de investimento futuro na implementação de práticas inclusivas (DIAS; CADIME, 2016). A investigação tem mostrado que a percepção de insucesso na implementação de práticas inclusivas tem um efeito nocivo sobre as atitudes dos professores relativamente à inclusão (AHMMED; SHARMA; DEPPELER, 2012), o que poderá explicar, em parte, os resultados observados no estudo no contexto pré-escolar português: dado que a extensão formal da educação especial ao pré-escolar é relativamente recente, os educadores podem ter formação e experiência limitada no que respeita à aplicação dos princípios da educação inclusiva em sala de aula. Para além disso, os resultados obtidos noutros estudos realizados em contexto pré-escolar sugerem que simplesmente fornecer formação e informação é insuficiente para garantir uma visão positiva da inclusão, e que a liderança da escola e o apoio governamental têm um papel bastante importante na promoção da eficácia dos professores para a inclusão (LEE et al., 2014). 


\section{0 presente estudo 3}

A investigação tem demonstrado que, apesar de as percepções dos professores, relativamente à inclusão na sala de aula regular, parecerem ser, em geral, positivas, é clara a existência de preocupações associadas à mesma, nomeadamente no que respeita à suficiência de recursos, conhecimentos e competências para lidar com as necessidades próprias dos alunos com necessidades educativas especiais. Perante a escassez de estudos sobre este tópico na educação pré-escolar, o presente trabalho faz uma análise exaustiva das percepções dos educadores de infância acerca da inclusão nas escolas do pré-escolar, explorando variáveis pessoais e profissionais que possam contribuir para explicar essas percepções.

\section{Método}

\subsection{Amostra}

Participaram neste estudo 118 educadores de infância portugueses, dos quais 116 eram do sexo feminino (98,3\%), sendo apenas 2 do sexo masculino $(1,7 \%)$. Relativamente à idade, $22.9 \%$ tinham entre 22 e 31 anos, 50,0\% tinham entre 32 e 41 anos, $21,2 \%$ tinham idade compreendida entre os 42 e os 51 anos e apenas $5,9 \%$ tinham idade superior. Metade da amostra era proveniente da zona norte de Portugal, $33,1 \%$ da zona centro e apenas $13,6 \%$ da zona sul do país (sem informação sobre a zona geográfica de proveniência de 3,4\% da amostra). No Quadro 1 é apresentada a caracterização da amostra em função do setor, habilitações académicas, experiência de trabalho como educador e experiência de trabalho com crianças com necessidades especiais. Relativamente ao setor de trabalho, quase metade dos participantes trabalhava numa instituição da rede pública de ensino, sendo que os restantes provinham de instituições de ensino privadas $(33,1 \%)$ e de estabelecimentos de ensino cooperativo $(21,2 \%)$. Relativamente às habilitações académicas, a grande maioria dos educadores possuía apenas licenciatura ou bacharelato, e, somente $8,5 \%$ da amostra tinha qualificações académicas de nível pós-graduado (pós-graduação ou mestrado). A maioria dos participantes tinha mais de 10 anos de experiência como educador (ver Quadro 1). A amostra era relativamente heterogénea, no que concerne ao número de anos de experiência na aplicação de práticas inclusivas: $11,9 \%$ reportou não ter nenhuma experiência, $11 \%$ relatou estar a aplicar este tipo de práticas há apenas um ano, $7,6 \%$ relatou ter $2-3$ anos de experiência, $8,5 \%$ entre $4-5$ anos de experiência e $52,5 \%$ relatou aplicar este tipo de práticas há mais de 6 anos. Relativamente à percentagem do dia que cada educador dedicava ao trabalho direto com crianças

Trabalho financiado ao abrigo do PEsr-OE/FIL/UI0683/2014 e pela bolsa SFRH/BPD/102549/2014 da Fundação para a Ciência e Tecnologia. 
que se beneficiavam de serviços de educação especial, verificou-se, igualmente, bastante heterogeneidade (ver Quadro 1).

\section{Instrumentos}

\subsection{Questionário sociodemográfico}

Foi utilizado um questionário sociodemográfico que permitiu recolher dados sobre os participantes, nomeadamente o género, idade, área geográfica e tipo de estabelecimento de ensino em que lecionava.

\subsection{Inventário de Inclusão}

O Inventário de Inclusão (The Inclusion Inventory; BECKER; ROBERTS; DUMAS, 2000) é um instrumento desenvolvido para avaliar as percepções de professores e educadores sobre as práticas inclusivas que são utilizadas na sua escola ou instituição.

Quadro 1. Caracterização da amostra em função do setor de trabalho, habilitaç̃̃es académicas e experiência de trabalho.

\begin{tabular}{|l|c|c|}
\hline Variáveis & $\mathbf{N}$ & $\%$ \\
\hline Setor & & \\
\hline Ensino público & 54 & 45,8 \\
\hline Ensino privado & 39 & 33,1 \\
\hline Ensino cooperativo & 25 & 21,2 \\
\hline Habilitações académicas & & \\
\hline Graduação & 108 & 91,5 \\
\hline Pós-graduação & 10 & 8,5 \\
\hline Experiência como educador & & \\
\hline$<5$ anos & 24 & 20,3 \\
\hline 6 a 10 anos & 19 & 16,1 \\
\hline$>10$ anos & 75 & 63,6 \\
\hline Experiência na aplicação de práticas inclusivas & & \\
\hline Nenhuma & 14 & 11,9 \\
\hline$<1$ ano & 13 & 11,0 \\
\hline 2 a 3 anos & 9 & 7,6 \\
\hline 4 a 5 anos & 10 & 8,5 \\
\hline$>6$ anos & 62 & 52,5 \\
\hline Sem informação & 10 & 8,5 \\
\hline Duração do trabalho diário com crianças com necessidades educativas especiais & & \\
\hline Nenhum & 36 & 30,5 \\
\hline Meio-dia ou menos & 32 & 27,1 \\
\hline Mais de meio dia & 37 & 31,4 \\
\hline Sem informação & 13 & 11,0 \\
\hline
\end{tabular}

Fonte: Elaborado pelos autores (2016). 
O instrumento é constituído por uma primeira seção de recolha de informação de background como o papel do docente na escola, a experiência no ensino e a experiência prévia e atual com práticas inclusivas, bem como por 68 outros itens agrupados em sete subescalas: (1) planeamento de práticas inclusivas - avalia a adequação do planeamento e desenvolvimento profissional em termos de inclusão (17 itens); (2) apoio nas práticas inclusivas - explora o apoio percebido para a aplicação de práticas inclusivas, bem como as oportunidades para o planeamento e a adequação de recursos (5 itens); (3) utilização de práticas inclusivas - avalia as práticas de educação inclusiva que os respondentes já adotaram (10 itens), (4) implementação de práticas inclusivas - explora quão frequentemente os alunos da educação especial se encontram envolvidos nas várias atividades da instituição de ensino e interagem satisfatoriamente com os restantes alunos (14 itens), (5) crença acerca de práticas inclusivas - avalia a concordância dos respondentes com várias afirmações sobre educação inclusiva, como, por exemplo, se os alunos do ensino regular beneficiam-se socialmente com a adoção de práticas inclusivas (11 itens), (6) efeito das práticas inclusivas - explora se os respondentes acreditam que os alunos que se beneficiam de educação especial em contextos inclusivos têm resultados piores, melhores ou semelhantes, em termos de desempenho académico, competências sociais, autoestima e assiduidade do que o que teriam em contextos tradicionais de educação especial (4 itens), (7) prática educativa na sala - avalia quão frequentemente os respondentes utilizam várias estratégias de ensino tais como a aprendizagem cooperativa ( 7 itens).

Em cada item os participantes devem dar uma resposta utilizando uma escala likert de três, quatro ou cinco pontos. Os escores totais para cada subescala são obtidos somando a resposta a cada item e dividindo-a pelo número de itens respondidos. Os valores de consistência interna da versão original foram muito elevados (Planeamento: $\alpha=0,96$; Apoio: $\alpha=0,85$; Utilização: $\alpha=0,89$; Implementação: $\alpha=0,86$; Crenças: $\alpha=0,72$; Efeitos: $\alpha=0,82$; Práticas educativas em sala de aula: $\alpha=0,81)$ e esta apresenta bons indicadores de validade por referência a critério (Becker; Roberts; Dumas, 2000).

\section{Procedimento}

Utilizou-se uma técnica de amostragem não probabilística para a recolha de dados. Começou-se por solicitar a autorização para a recolha de dados aos diretores de instituições de ensino com valência de jardim de infância da zona Norte, Centro e Sul de Portugal. Após a obtenção do consentimento destes, os coordenadores de departamento foram convidados a colaborar, motivando os educadores de infância para participarem do estudo, distribuindo os questionários na sua versão impressa e recolhendo os questionários após o seu preenchimento. Garantiu-se o 
anonimato e a natureza voluntária da participação, tendo todos os participantes assinado uma folha de consentimento informado antes do preenchimento dos questionários.

\subsection{Análise de dados}

Uma vez recolhidos, os dados foram informatizados e analisados com o programa de tratamento estatístico IBM SPSS Statistics 23. Calculou-se o Alfa de Cronbach para avaliação da consistência interna dos itens em cada uma das subescalas do instrumento de avaliação das práticas inclusivas. Considerou-se o valor mínimo de .70 como indicador de uma boa consistência interna dos itens (GEORGE; MALLERY, 2002).

Calculou-se a média e desvio padrão, bem como os valores de assimetria e curtose, para cada variável. Face aos elevados valores de assimetria e curtose e dado que os testes de normalidade de Kolmogorov-Smirnov se revelaram significativos, utilizou-se estatística inferencial de tipo não paramétrico. Para testar a existência de diferenças, em função das habilitações literárias (bacharelato/licenciatura versus pós-graduação/mestrado) nas sete dimensões, recorreu-se ao teste não paramétrico de Mann-Whitney.

Para avaliar a existência de uma associação entre as sete dimensões das percepções sobre as práticas inclusivas e a idade, experiência docente, anos de experiência na aplicação de práticas inclusivas e percentagem do dia dedicada a trabalhar com crianças que recebem serviços de educação especial, recorreu-se ao cálculo do coeficiente de correlação de Spearman.

\section{Resultados}

No Quadro 2, apresentam-se os valores do alpha de Cronbach para cada subescala do Inventário de Inclusão. Em todas as subescalas, os valores obtidos foram bastante superiores ao valor de referência de 0,70 , indicando uma elevada consistência interna dos itens.

No Quadro 3, apresenta-se a estatística descritiva para cada dimensão das percepções sobre a implementação de práticas inclusivas, bem como as correlações (Spearman) entre as diferentes subescalas. Observaram-se distribuições bastante assimétricas, especialmente nas subescalas de implementação e efeitos das práticas educativas, com todos os escores das sete subescalas a apresentarem violações à normalidade. Encontraram-se correlações significativas entre a pontuação na subescala de planeamento e as pontuações nas subescalas de apoio, 
Quadro 2. Consistência interna dos itens nas sete subescalas.

\begin{tabular}{|l|c|c|}
\hline Variáveis & $\mathbf{N}^{\circ}$ itens & Alpha de Cronbach \\
\hline Planeamento & 17 & 0,94 \\
\hline Apoio & 5 & 0,85 \\
\hline Utilização & 10 & 0,85 \\
\hline Implementação & 14 & 0,85 \\
\hline Crenças & 11 & 0,69 \\
\hline Efeitos & 4 & 0,74 \\
\hline Práticas educativas na sala & 7 & 0,83 \\
\hline
\end{tabular}

Fonte: Elaborado pelos autores (2016).

utilização e implementação das práticas inclusivas, e práticas educativas usadas em sala de aula. Encontrou-se igualmente uma correlação significativa entre a implementação de práticas inclusivas e uma pontuação mais elevada em termos de apoio e utilização anterior de práticas inclusivas. Por fim, verificou-se que maior percepção de efeitos positivos das práticas inclusivas estava associada a maior nível de recurso a práticas educativas como a aprendizagem cooperativa em sala de aula.

No Quadro 4, apresentam-se os resultados do teste de Mann-Whitney (U) para testar a existência de diferenças em função das habilitações literárias. Não se verificaram diferenças entre os grupos de educadores com diferentes níveis de formação a nível de nenhuma das sete dimensões das práticas inclusivas. Saliente-se, no entanto que o resultado do teste para a variável planeamento foi marginalmente significativo $(\mathrm{p}=0,051)$, tendo os educadores com formação de nível pós-graduado relatado maior planeamento de práticas inclusivas do que aqueles sem formação pós-graduada.

No Quadro 5, apresentam-se os coeficientes de correlação de Spearman entre os escores obtidos para as sete dimensões das práticas inclusivas e a idade, experiência docente dos educadores, anos de experiência na aplicação de práticas inclusivas e percentagem do dia dedicada a trabalhar com crianças que recebem serviços de educação especial.

Os resultados apontaram uma relação significativa e inversa entre a dimensão de apoio e o tempo despendido com crianças com necessidades educativas especiais, sugerindo que menor percentagem do dia despendida a trabalhar com crianças que recebem serviços da educação especial se encontra associada a maior percepção de apoio nas práticas inclusivas. 

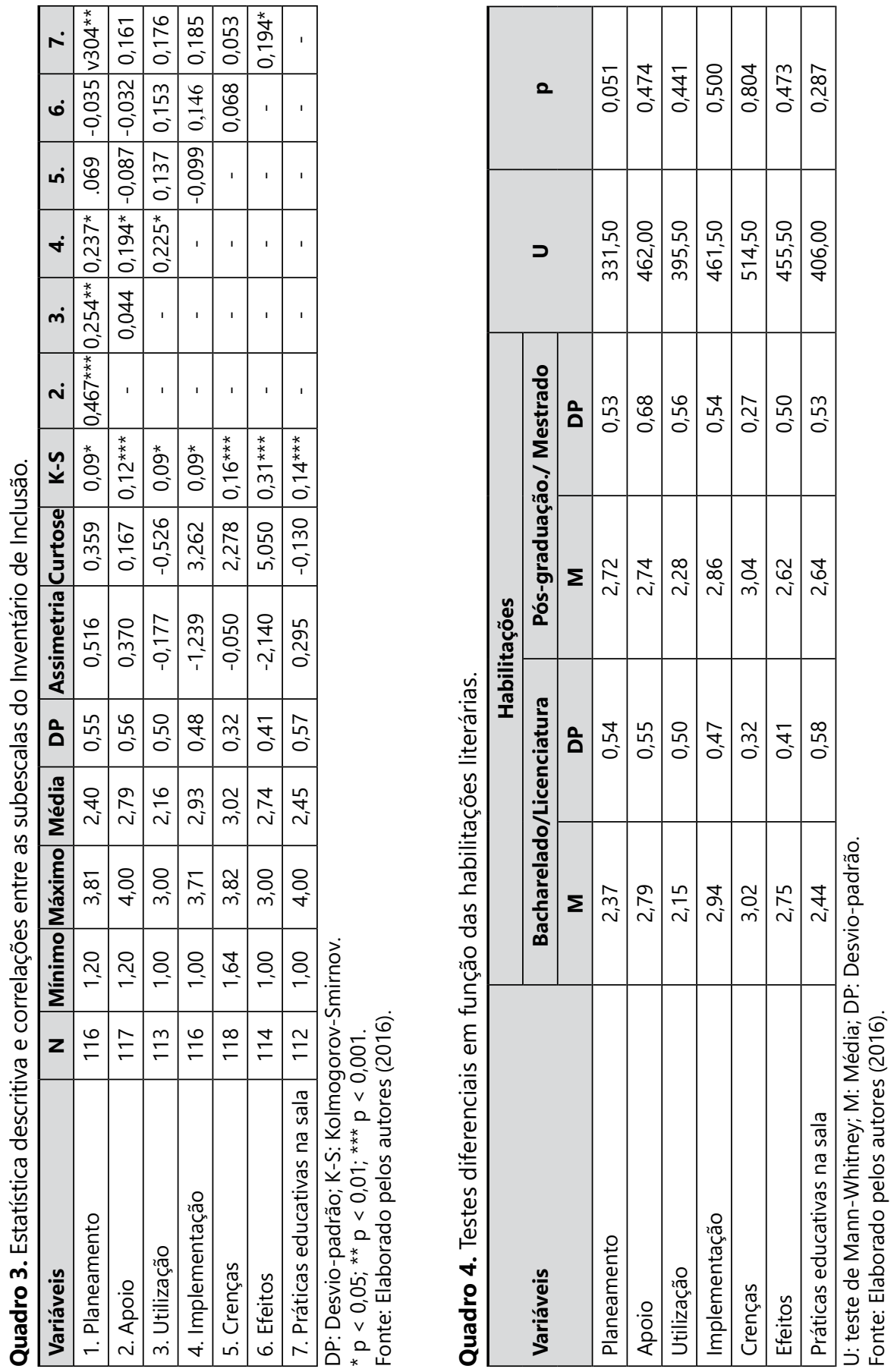
Encontraram-se também coeficientes de correlação significativos e negativos entre as crenças sobre as práticas inclusivas, a idade e os anos de experiência docente e na aplicação de práticas inclusivas, indicando que educadores mais novos, com menos anos de experiência e com menos tempo de experiência na aplicação de práticas inclusivas têm pontuações mais elevadas em termos de crenças sobre a inclusão.

Todavia, observou-se também uma correlação positiva entre os escores na subescala de efeitos das práticas educativas e a idade e percentagem de tempo dedicada a trabalhar com crianças que recebem serviços de Educação Especial. Este resultado indica que educadores mais velhos e educadores que passam mais tempo a trabalhar com crianças com necessidades educativas especiais percecionam melhores outcomes em termos de desempenho académico, competências sociais, autoestima e assiduidade nas crianças que usufruem de serviços de educação especial.

Todas as restantes correlações foram não significativas.

\section{Discussão e conclusões}

O objetivo geral deste estudo foi explorar quais as percepções que os educadores da educação pré-escolar possuíam sobre a implementação da inclusão nas suas escolas e investigar se as características pessoais e profissionais destes educadores se relacionavam com as suas percepções. Os resultados da análise da fidelidade do principal instrumento utilizado para a recolha de dados - Inventário de Inclusão

Quadro 5. Matriz de correlações (Spearman) entre as dimensões das práticas inclusivas e a idade, experiência docente, experiência na aplicação de práticas inclusivas e trabalho diário com crianças com necessidades educativas especiais.

\begin{tabular}{|l|c|c|c|c|}
\hline Variáveis & Idade & $\begin{array}{c}\text { Experiência } \\
\text { docente } \\
\text { (anos) }\end{array}$ & $\begin{array}{c}\text { Experiência } \\
\text { PI (anos) }\end{array}$ & $\begin{array}{c}\text { Trab. diário } \\
\text { crianças NEE }\end{array}$ \\
\hline Planeamento & 0,054 & 0 & 0,001 & 0,058 \\
\hline Apoio & $-0,098$ & $-0,029$ & 0,048 & $-0,232^{*}$ \\
\hline Utilização & 0,136 & 0,118 & 0,075 & 0,170 \\
\hline Implementação & 0,162 & 0,163 & 0,072 & 0,186 \\
\hline Crenças & $-0,298^{\star *}$ & $-0,257^{\star *}$ & $-0,230^{*}$ & 0,095 \\
\hline Efeitos & $0,232^{*}$ & 0,122 & $-0,033$ & $0,197^{*}$ \\
\hline Práticas educativas na sala & 0,055 & 0,102 & $-0,016$ & 0,138 \\
\hline
\end{tabular}

PI: Práticas inclusivas; NEE: Necessidades educativas especiais.

${ }^{*} \mathrm{p}<0,05 ;{ }^{* *} \mathrm{p}<0,01$.

Fonte: Elaborado pelos autores (2016). 
(BECKER; ROBERTS; DUMAS, 2000) - mostraram que todas as subescalas apresentavam indicadores adequados de consistência interna, embora a subescala de crenças acerca de práticas inclusivas apresentasse o valor menos elevado. Este indicador fornece indicações da solidez e robustez do instrumento de recolha de dados utilizado.

Os resultados do presente estudo mostraram ainda que a implementação de práticas inclusivas - avaliada por questões que exploram quão frequentemente os alunos da educação especial se encontram envolvidos nas várias atividades da instituição de ensino e interagem satisfatoriamente com os restantes alunos - se encontra positiva e significativamente correlacionada com níveis mais elevados de planeamento, maior percepção de apoio percebido para a aplicação de práticas inclusivas, incluindo adequação de recursos, e utilização anterior de práticas inclusivas. Resultados semelhantes têm sido encontrados em estudos com professores de outros níveis de ensino. Relativamente ao planeamento e ao apoio percebido, estudos recentes têm demostrado que projetos de mentoria, a cooperação com os colegas e a colaboração entre profissionais especializados são essenciais para uma prática pedagógica inclusiva, com benefícios para todos os alunos em termos de competências e de envolvimento entre pares (FLEM; KELLER, 2000; LINDQVIST; NILHOLM, 2014; TJERNBERG; MATTSON, 2014). O apoio percebido para a aplicação de práticas inclusivas e as oportunidades para o planeamento e a adequação de recursos têm sido também fatores constantemente sinalizados pelos professores como determinantes para a eficácia das suas práticas (HORNE; TIMMONS, 2009; BALLHYSA; FLAGLER, 2011). Um resultado curioso, obtido no presente estudo, foi o de que menor percentagem do dia despendida a trabalhar com crianças que recebem serviços da educação especial se encontra associada à maior percepção de apoio nas práticas inclusivas. Este resultado pode indicar alguma difusão da responsabilidade do trabalho com os alunos com necessidades educativas especiais. Por outras palavras, é possível que os educadores provenientes de escolas que disponibilizam maior número de recursos humanos especializados trabalhem durante menos tempo diretamente com as crianças com necessidades especiais porque, em parte do tempo letivo, a responsabilidade pelo trabalho com estas crianças é atribuída a outros profissionais (e.g.: professores de Educação Especial). Esta possível explicação para o resultado observado deverá ser explorada em estudos futuros, podendo ser indicativa de alguma necessidade de melhor articulação para fazer face a este desafio recente.

Não se observaram diferenças entre os grupos de educadores com diferentes níveis de formação em termos de nenhuma das dimensões avaliadas, embora os resultados tenham sido marginalmente significativos para as questões relacionadas com o planeamento, com os educadores com formação de nível pós-graduado 
a relatarem maiores níveis de planeamento e desenvolvimento profissional em termos de inclusão. Uma das limitações deste estudo prende-se com o facto de não ter sido avaliado se a formação pós-graduada que os educadores realizaram era ou não focada em questões relacionadas com necessidades educativas especiais ou educação especial. A possível frequência deste tipo de formação pósgraduada por parte de uma percentagem da amostra poderia explicar parcialmente este resultado. Resultados da investigação anterior (ROMI; LEYSER, 2006) têm demonstrado que a frequência deste tipo de formação, quer seja de nível inicial ou contínuo, poderá ter efeitos positivos nas práticas de inclusão que os professores adotam, ao fomentar a sua autoeficácia e atitudes mais positivas face à inclusão. A necessidade de formação especializada para trabalhar com crianças com necessidades educativas especiais é consistentemente apontada como uma das necessidades mais referidas por professores de vários níveis de ensino (KOUTROUBA; VAMVAKARI; THEODOROPOULOS, 2008).

Os resultados do presente estudo mostraram também que educadores mais novos, com menos anos de experiência e com menos tempo de experiência na aplicação de práticas inclusivas, têm pontuações mais elevadas em termos de crenças sobre a inclusão. Se bem que menos explorados junto de educadores, estes resultados estão em linha com estudos anteriores, que parecem sugerir que os professores mais velhos tendem a apresentar percepções menos favoráveis em relação à inclusão (e.g.: STOIBER; GETTINGER; GOETZ, 1998; VAZ et al., 2015). Todavia, os resultados deste estudo indicaram também que educadores mais velhos e educadores que passam mais tempo a trabalhar com crianças com necessidades educativas especiais percecionam melhores resultados em termos de desempenho académico, competências sociais, autoestima e assiduidade nas crianças que usufruem serviços de educação especial. Estes dados parecem ir ao encontro dos obtidos em estudos que apontam o papel da experiência e o contacto com crianças com necessidades especiais como forte preditor das percepções e do sentido de eficácia.

$\mathrm{Na}$ integração dos dados com a literatura, percebe-se que muito há ainda a fazer (HENNINGER IV; GUPTA, 2014). Os desafios atuais implicam uma aliança entre diferentes atores, das autoridades educativas, às universidades e escolas, no sentido de aprofundar esta cultura inclusiva. Parece necessária a criação de oportunidades de formação específica neste domínio, aprofundando a relação entre os desenvolvimentos teóricos e da investigação, com as práticas dos educadores no seu trabalho quotidiano (DUCHNOWSKI et al., 2006; COOK; COOK; LANDRUM, 2013). Importa, portanto, promover nos programas de formação um maior contacto com a literatura de referência, permitindo compreender melhor a investigação, explorar a sua importância e ajudar a perceber como aplicar os 
resultados dos contextos controlados para o terreno (JONES, 2009). Para isso, parece-nos essencial ouvir os educadores e os seus contributos para a melhoria das suas práticas (PAULINO; CÔCO, 2016).

Apesar dos contributos do presente trabalho, não podemos deixar de apontar algumas limitações que devem ser tidas em conta na apreciação do grau de generalização que pode ser feito dos resultados obtidos. Desde logo, uma limitação relacionada com a metodologia utilizada (quantitativa e baseada em questionários de autorrelato): embora esta permita captar uma figura precisa $\mathrm{e}$ abrangente da realidade, as respostas dos participantes podem sofrer um efeito de desejabilidade social. A amostra tem uma dimensão razoável, contudo, será ambicioso considerar que representa fielmente a realidade dos educadores de infância, pelo que estudos com amostras maiores e representativas dos educadores portugueses serão um desafio no futuro.

\section{Considerações finais}

Quaisquer tentativas de promoção de práticas mais inclusivas, incluindo políticas educativas de maior ou menor alcance, devem ter em consideração as percepções, atitudes e pontos de vista dos professores tanto do ensino regular, como da educação especial. Decorrentes da avaliação destas percepções, os resultados deste estudo sublinham a necessidade de as diferentes estruturas educativas proporcionarem meios, recursos e suporte à implementação de práticas educativas inclusivas junto dos profissionais que trabalham neste nível de ensino, dada a ainda parca experiência destes na gestão de ambientes inclusivos, fruto das recentes alterações legislativas e estruturais. Para além disso, parece existir um campo fértil para o desenvolvimento e avaliação de ações de formação contínua, no sentido de promover conhecimentos adequados sobre as necessidades educativas especiais, assim como das estratégias empiricamente validadas para maximizar o potencial de desenvolvimento das crianças nesta faixa etária. Num contexto em que ainda se está a organizar também o sistema de intervenção precoce, outras linhas de investigação e de colaboração entre universidade e as instituições, parecem emergir. 


\section{Preschool teachers' perceptions about inclusion in preschool education: the role of experience and qualifications}

\section{Abstract}

In Portugal, special education services were recently extended to preschool education. The studies that evaluate the perceptions of the preschool teachers about inclusive practices are also few. In this study we aim to evaluate the perceptions of preschool teachers about inclusion. A sample of 118 Portuguese preschool teachers was used. The Inclusion Inventory was administrated. The results indicate that the implementation of inclusive practices is related to perceived support and previous use of inclusive practices. Younger teachers, with less teaching experience and less time implementing inclusive practices have more beliefs about inclusion. Older professionals and teachers who spend more time working with children with special needs perceive better effects of inclusion in children. These results are discussed considering the existing literature, and implications for educational practice and research are presented.

Keywords: Inclusion. Preschool. Perceptions. Preschool teachers.

\section{Las percepciones de los educadores hacia la inclusión en la educación preescolar: el rol de la experiencia y de las calificaciones}

\section{Resumen}

En Portugal, la extensión de los servicios de educación especial a la educación preescolar es relativamente reciente. También son pocos los estudios que evalúan las percepciones de los maestros de la educación preescolar en las prácticas inclusivas. En este trabajo nos proponemos evaluar las percepciones de los maestros de la educación preescolar acerca de la inclusión. Se utilizó una muestra de 118 educadores portugueses, los cuales respondieran al inventario de inclusión. Los resultados indican que las prácticas inclusivas están relacionadas con la percepción de apoyo y el uso previo de las prácticas inclusivas. Los educadores más jóvenes, con menos experiencia de trabajo y menos experiencia lectiva en la aplicación de prácticas inclusivas, tienen más creencias acerca de la inclusión. Educadores con más edad y con más tiempo de servicio con niños con necesidades educativas especiales perciben mejores efectos de su inclusión. Estos resultados se discuten a la luz de la literatura existente, presentando también implicaciones para la práctica educativa y la investigación.

Palabras claves: Inclusión. Educación preescolar. Percepciones. Maestros de educación preescolar. 


\section{Referências}

AHMMED, M.; SHARMA, U.; DEPPELER, J. Variables affecting teachers' attitudes towards inclusive education in Bangladesh. Journal of Research in Special Educational Needs, v. 12, n. 3, p. 132-140, Jul. 2012.

AVRAMIDIS, E.; BAYLISS, P.; BURDEN, R. A survey into mainstream teachers' attitudes towards the inclusion of children with special educational needs in the ordinary school in one local education authority. Educational Psychology, v. 20, n. 2, p. 191-211, 2000.

AVRAMIDIS, E.; KALYVA, E. The influence of teaching experience and professional development on Greek teachers' attitudes towards inclusion. European Journal of Special Needs Education, v. 22, n. 4, p. 367-389, 2007.

AVRAMIDIS, E.; NORWICH, B. Teachers' attitudes towards integration/ inclusion: a review of the literature. European Journal of Special Needs Education, v. 17, n. 2, p. 129-147, 2002.

BAIRRÃO, J.; VASCONCELOS, T. A educação pré-escolar em Portugal: contributos para uma perspectiva histórica. Inovação, v. 10, p. 7-19, 1997.

BALLHYSA, N.; FLAGLER, M. A teachers' perspective of inclusive education for students with special needs in a model demonstration project. Academicus International Scientific Journal, n. 3, p. 121-133, 2011.

BECKER, H.; ROBERTS, G.; DUMAS, S. The inclusion inventory: A tool to assess perceptions of the implementation of inclusive educational practices. Special Services in Schools, v. 16, n. 1-2, p. 57-72, 2000.

DE BOER, A.; PIJL, S. J.; MINNAERT, A. Regular primary schoolteachers' attitudes towards inclusive education: A review of the literature. International Journal of Inclusive Education, v. 15, n. 3, p. 331-353, 2011.

DE BOER, A.; PIJL, S. J.; MINNAERT, A.; POST, W. Evaluating the effectiveness of an intervention program to influence attitudes of students towards peers with disabilities. Journal of Autism and Developmental Disorders, v. 44, n 3, p. 572-583, March. 2014.

CALHEIROS, M. M.; GRAÇA, J.; PATRÍCIO, J. N. From assessing needs to designing and evaluating programs: Case study of a family support program in Portugal. Children and Youth Services Review, v. 36, p. 170-178, January 2014. 
COOK, B. G.; COOK, L.; LANDRUM, T. J. Moving research into practice: Can we make dissemination stick? Exceptional Children, v. 79, n. 2, p. 163-180, 2013.

DIAMOND, K. E. et al. Synthesis of IES Research on Early Intervention and Early Childhood Education (NCSER 2013-3001). Washington, DC: National Center for Special Education Research, Institute of Education Sciences, U.S. Department of Education, 2013.

DIAS, P. C. For a qualitative leap in inclusion: Illusions and delusions from teacher's perspective. Procedia - Social and Behavioral Sciences, v. 191, p. 1119-1123, June 2015.

DIAS, P. C.; CADIME, I. Effects of personal and professional factors on teachers' attitudes towards inclusion in preschool. European Journal of Special Needs Education, v. 31, n. 1, p. 111-123, 2016.

DIREÇÃO-GERAL DE ESTATÍSTICAS DA EDUCAÇÃO E CIÊNCIA DGEEC. Educação em números: Portugal - 2015. Lisboa: DGEEC, 2015.

DUCHNOWSKI, A.J. et al. Increasing the use of evidence-based strategies by special education teachers: A collaborative approach. Teaching \& Teacher Education: An International Journal of Research and Studies, v. 22, n. 7, p. 838-847, 2006.

FLEM, A.; KELLER, C. Inclusion in Norway: A study of ideology in practice. European Journal of Special Needs Education, v. 15, n. 2, p. 188-205, 2000.

GEORGE, D.; MALLERY, P. SPSS for Windows step by step: A simple guide and reference. 4th ed. Boston: Allyn \& Bacon, 2002.

HEIMAN, T. TEachers coping with changes: Including students with disabilities in mainstream classes: An international view. International Journal of Special Education, v. 19, n. 2, p. 91-103, 2004.

HENNINGER IV, W. R.; GUPTA, S. S. How do children benefit from inclusion? In: GUPTA, S. S.; HENNINGER IV, W. R.; VINH, M. E. (Ed.), First steps to preschool inclusion: How to jumpstart your programwide plan. Baltimore: Brookes Publishing, 2014. p. 33-57.

HORNE, P.E.; TIMMONS, V. Making it work: teachers' perspectives on inclusion. International Journal of Inclusive Education, v. 13, n. 3, p. 273-286, 2009. 
HUNDERT, J.; ROWE, S.; HARRISON, E. The combined effects of social script training and peer buddies on generalized peer interaction of children with ASD in inclusive classrooms. Focus on Autism and Other Developmental Disabilities, v. 29, n. 4, p. 206-215, December 2014.

JONES, M. L. A study of novice special educators' views of evidence-based practices. Teacher Education and Special Education, v. 32, n. 2, p. 101-120, 2009.

KOUTROUBA, K.; VAMVAKARI, M.; THEODOROPOULOS, H. SEN students' inclusion in Greece: factors influencing Greek teachers' stance. European Journal of Special Needs Education, v. 23, n. 4, p. 413-421, 2008.

LEE, F. L. et al. What predicts teachers' acceptance of students with special educational needs in kindergarten? Australian Journal of Educational \& Developmental Psychology, v. 14, p. 60-70, 2014.

LINDQVIST, G.; NILHOLM, C. Promoting inclusion? 'Inclusive' and effective head teachers' descriptions of their work. European Journal of Special Needs Education, v. 29, n. 1, p. 74-90, 2014.

LOREMAN, T.; FORLIN, C.; SHARMA, U. An international comparison of pre-service teacher attitudes towards inclusive education. Disability Studies Quarterly, v. 27, n. 4, 2007.

OGELMAN, H. G.; SECER, Z. The effect inclusive education practice during preschool has on the peer relations and social skills of 5-6-year olds with typical development. International Journal of Special Education, v. 27, n. 3, p. 169-175, 2012.

PAULINO, V. B. R.; CÔCO, V. Políticas públicas educacaionais: vozes que emerge no trabalho docente na Educação Infantil. Ensaio: Avaliação e Políticas Públicas em Educação, v. 24, n. 92, p. 697-718, 2016.

PEARS, K. C. et al. Improving child self-regulation and parenting in families of pre-kindergarten children with developmental disabilities and behavioral difficulties. Prevention Science, v. 16, n. 2, p. 222-232, 2014.

PORTUGAL. Lei no 319/1991. Estabelece o regime educativo especial aplicável aos alunos com necessidades educativas especiais. Diário da República, Portugal, de 23 ago. 1991. S.1, n. 193, p. 4389-4393.

. Lei n ${ }^{\circ}$ 5/97. Lei-Quadro da Educação Pré-Escolar. Diário da República, Portugal, de 10 fev. 1997a. Seção 1, n. 34, p. 670-673. 
PORTUGAL. Ministério da Educação. Orientações Curriculares para a Educação Pré- Escolar. Lisboa: Ministério de Educação/Departamento de Educação Básica, 1997b.

. Decreto-Lei n ${ }^{0} 3 / 2008$. Apoios especializados a prestar na educação préescolar e nos ensinos básico e secundário dos sectores público, particular e cooperativo. Diário da República, Portugal, de 7 jan. 2008. Seção 1, n. 4, p. 154-164.

PRINCE, E. J.; HADWIN, J. The role of a sense of school belonging in understanding the effectiveness of inclusion of children with special educational needs. International Journal of Inclusive Education, v. 17, n. 3, p. 238-262, 2013.

RODRIGUES, F. B. et al. Family-school cooperation in the context of inclusion of children with special educational needs. Procedia - Social and Behavioral Sciences, v. 171, p. 309-316, 2015.

ROMI, S.; LEYSER, Y. Exploring inclusion preservice training needs: a study of variables associated with attitudes and self-efficacy beliefs. European Journal of Special Needs Education, v. 21, n. 1, p. 85-105, 2006.

RUIJS, N. M.; PEETSMA, T. T. Effects of Inclusion on Students with and without Special Educational Needs Reviewed. Educational Research Review, v. 4, n. 2, p. 67-79, 2009.

SAINATO, D. M. et al. A comprehensive inclusion program for kindergarten children with autism spectrum disorder. Journal of Early Intervention, v. 37, n. 3, p. 208-225, 2015.

SÁNCHEZ, P. A.; ABELLÁN, C. M. A. Autoevaluación docente para la mejora de los procesos educativos en escuelas que caminan hacia la inclusión. Revista Colombiana de Educación, n. 67, p. 227-245, 2014.

STOIBER, K. C.; GETTINGER, M.; GOETZ, D. Exploring factors influencing parents' and early childhood practitioners' beliefs about inclusion. Early Childhood Research Quarterly, v. 13, n. 1, p. 107-124, 1998.

TJERNBERG C.; MATTSON, E. H. Inclusion in practice: a matter of school culture. European Journal of Special Needs Education, v. 29, n. 2, p. 247-256, 2014.

UNITED NATIONS EDUCATIONAL, SCIENTIFIC AND CULTURAL ORGANIZATION - UNESCO. Final Report - World Conference on Special Needs Education: Access and quality. Paris: UNESCO, 1994. 
VASCONCELOS, T. Early childhood education and equity issues in Portugal: A case study of four settings. Journal of Early Childhood Research, v. 3, n. 2, p. 127-148, 2005.

. A participatory process of developing a recommendation for the government about the education of children from birth to three years: the case of Portugal. International Journal of Early Years Education, v. 21, n. 2-3, 125-140, 2013.

VAZ, S. et al. Factors associated with primary school teachers' attitudes towards the inclusion of students with disabilities. PLoS One, v. 10, n. 8, p. e0137002, 2015.

WIENER, J.; TARDIF, C. Y. Social and emotional functioning of children with learning disabilities: Does special education placement make a difference?

Learning Disabilities Research and Practice, v. 19, n. 1, p. 20-32, February 2004.

\section{Informações dos autores}

Paulo César Azevedo Dias: Doutor. Professor Auxiliar da Faculdade de Filosofia e Ciências Sociais da Universidade Católica Portuguesa. Contato: pcdias@braga.ucp.pt

Irene Maria Dias Cadime: Doutora. Bolseira de Pós-Doutoramento no Centro de Investigação em Estudos da Criança da Universidade do Minho. Contato: irenecadime@ ie.uminho.pt 